\title{
The Design of Fingerprint Identification System based on Improved Binarization Algorithm
}

\author{
Zhu Heng-jun ${ }^{1}$, Guo Ying ${ }^{1}$, Zhang nan-nan ${ }^{1}$ and Liang Hong ${ }^{1}$ \\ Communication and Electronics Engineering Institute of Qiqihar University, \\ Heilongjiang Qiqihar 161006, China; \\ gulin9969@163.com
}

\begin{abstract}
Many domestic enterprises use punch machine for attendance management of coal mine workers, which leads to have some underground hidden danger and have difficulties to confirm the accident personnel and rescue them afterwards. This paper describes the design of a fingerprint recognition system for coal mine workers identity recognition, which can guarantee the safe production. This design which is based on minutiae fingerprint algorithm has been improved, using DSP technology to design a fingerprint identification module. The system uses TMS320VC5509 made by TI Corporation as the core processor, which can easily carry out fingerprint acquisition, segmentation and matching. The result of debugging shows that this system can operate stably and reliably, improving the accuracy and efficiency of the security check.
\end{abstract}

Keywords: mining management, fingerprint recognition, bnarization algorithm, optical fingerprint sensor, TMS320VC5509 signal processor

\section{Introduction}

As a kind of biometric recognition method, fingerprint recognition using the uniqueness of fingerprint is widely concerned [1]. In the fingerprint algorithm, a good fingerprint segmentation algorithm is very important for fingerprint recognition system. The fingerprint segmentation algorithm typically includes two categories: algorithm based on grey variance and fingerprint segmentation algorithm based on directivity diagram of fingerprint. The algorithm based on grey variance method is not suitable for the low contrast images or strong noise images. In the low contrast and high noise environment, the results of fingerprint image segmentation are often inaccurate. The fingerprint segmentation algorithm based on directivity diagram has higher requirements for the precision of directivity diagram in a fingerprint image. The effect of segmentation depends on the reliability of directivity diagram in a fingerprint image, and this method is not sensitive to the contrast of the image. To the discontinuous of the ridge and the single grey area, the segmentation algorithm based on directivity diagram is difficult to achieve satisfactory results.

This paper has improved the previous methods and puts forward a new method called comprehensive orientation segmentation and variance partitioning, improving the anti-interference ability of the image segmentation. At the same time, this paper also uses improved binarization algorithm based on the global iterative method. The software is programmed with $\mathrm{C}$ language which can realize fingerprint image acquisition, segmentation, binarization, thinning algorithm and extraction characteristic value of endpoint and bifurcation point to achieve fingerprint comparison and identification. The experimental 
results show that the false accept rate (FAR) and false rejection rate (FRR) are less than or equal to $1 \%$. It has the advantages of improving the accuracy of fingerprint identification. The speed of the system has raised and the result of the test is satisfactory, which shows that the system is suitable for identity recognition and confirmation of underground coal mine workers.

\section{The Structure of the Fingerprint Identification System}

In this design, the structure of the fingerprint recognition system consists of four parts: optical fingerprint sensor acquisition module, DSP processing module, MCU control module and LCD display module. The components of the system are shown in Figure 2.1.

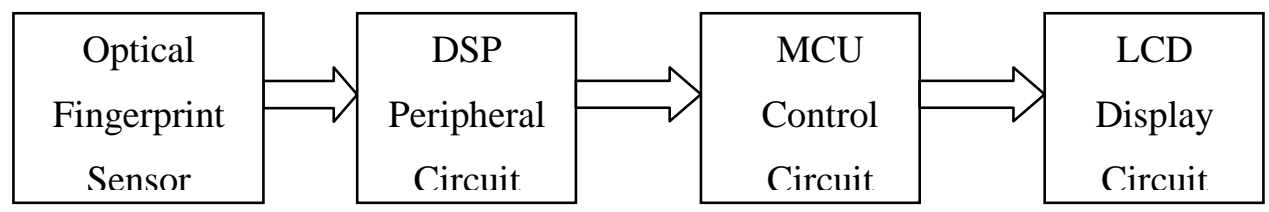

Figure 2.1. The Diagram of the System Structure

The hardware mainly is: optical fingerprint sensor, DSP data processing unit, peripheral control circuit and LCD display circuit. The structure of the DSP peripheral circuit is shown in Figure 2.2.

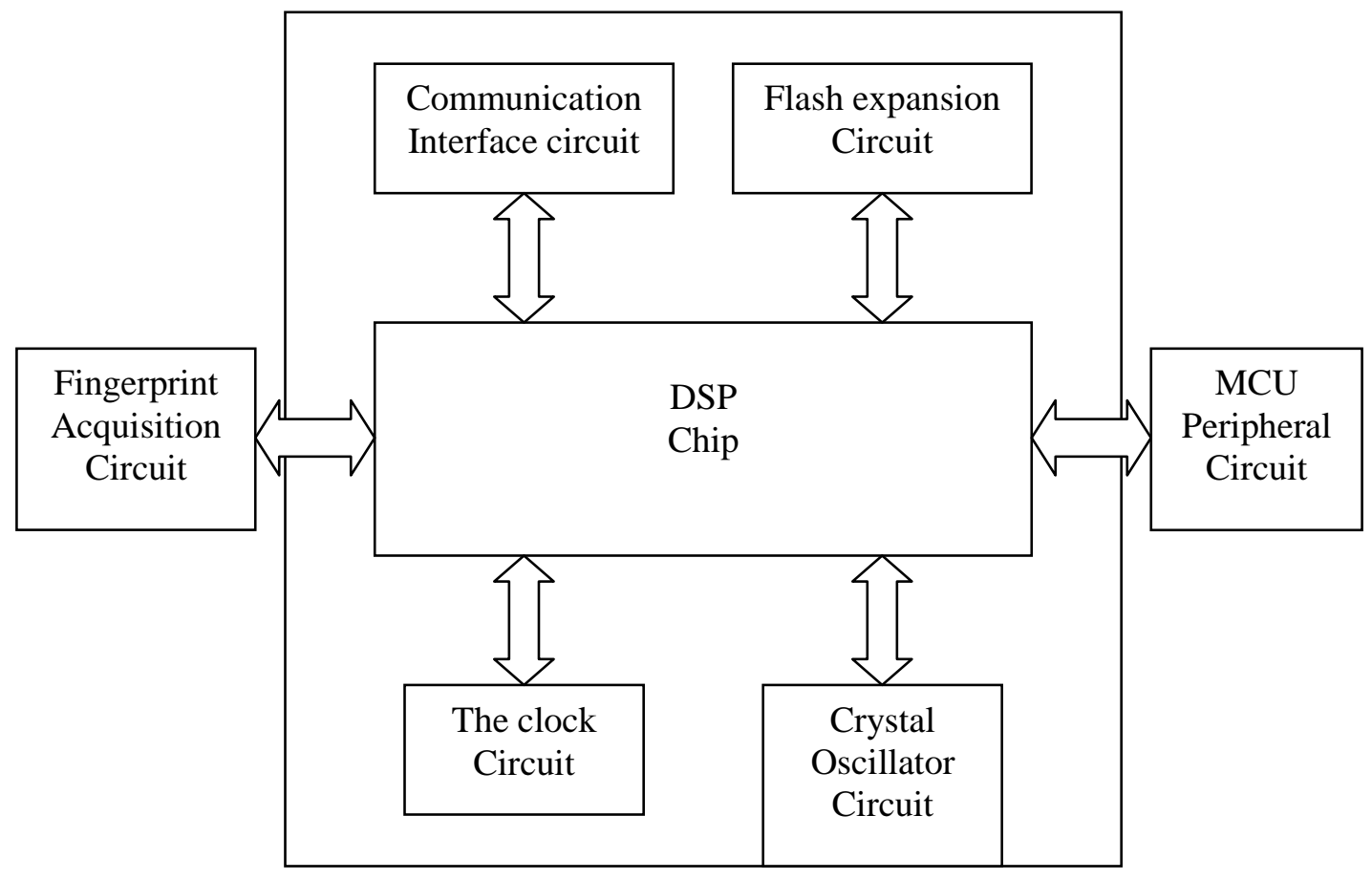

Figure 2.2. DSP Peripheral Circuit

According to the requirement of the system, DSP uses MTS320VC5509 chip made by TI Corporation. Its dominant frequency can reach $144 \mathrm{MHz}$. The instruction cycle is $6.9 \mathrm{~ns}$, and the chip has eight buses. It is produced by TI Company and is a fixed-point digital signal processor which has a competitive price. The MCU uses STC89C52 chip and the display 
module uses LCD12864 chip. We can store the data of fingerprint using large capacity flash memory. Using RS232 interface communicates with the computer to realize data transmission between the device and the computer.

The fingerprint image acquisition module uses optical fingerprint sensor called GC0303. GC0303 is a CMOS image sensor made by gcore Inc. The chip has a strong antistatic ability and stability. You can control the output of full frame through a two-wire serial communication port or use the way of sampling and window cutting to achieve the output of various kinds of image format. You can also control the quality or format of the image by setting register [2].

The overall design scheme of the software system includes: initialization program design, fingerprint identification program design and LCD display program design. Basic framework of the fingerprint recognition in this design is shown in Figure 2.3.

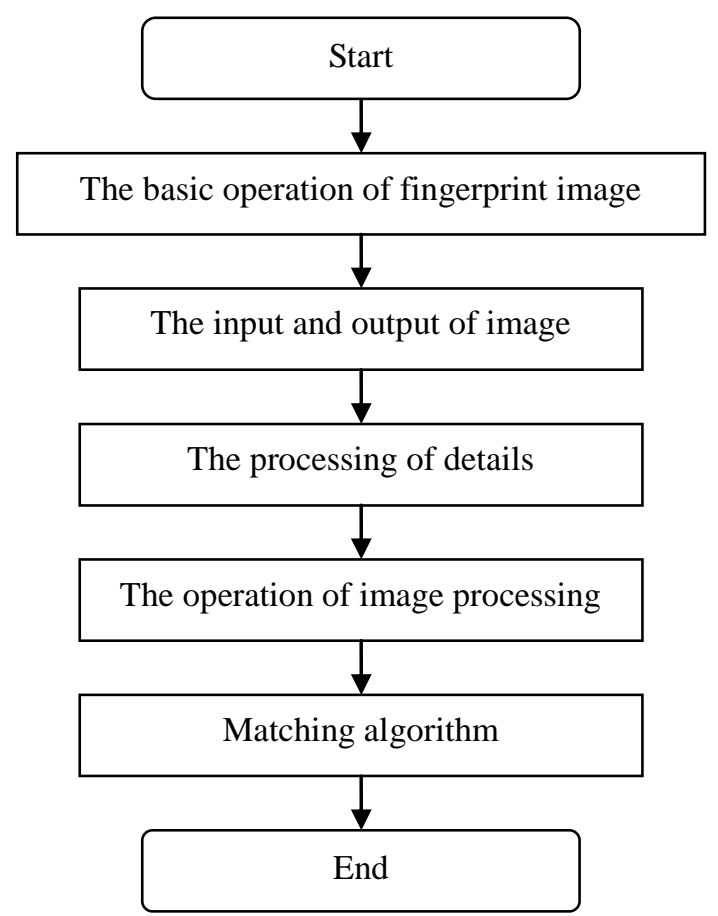

Figure 2.3. The Basic Block Diagram of Fingerprint Recognition

\section{The Design of Fingerprint Recognition Algorithm}

With the whole fingerprint recognition system, the fingerprint identification is generally divided into the following three steps: fingerprint image preprocessing, feature points extraction and matching. The fingerprint image preprocessing mainly has the following steps: Image filtering enhancement, normalization, binarization and thinning. Flow chart of the system is shown in Figure 3. 


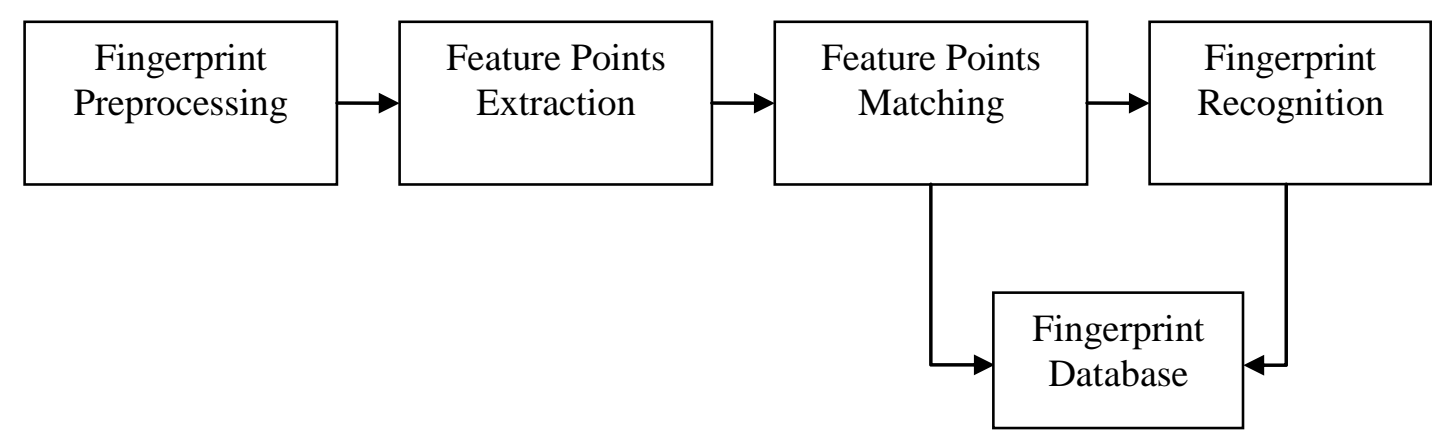

Figure 3. Flow Diagram of the System

\subsection{Fingerprint Image Segmentation}

A good fingerprint segmentation algorithm should have the following characteristics: be not sensitive to the gray distribution of fingerprint image, can detect the effective area of the relatively large noise, and can perform effective segmentation of the residual fingerprint. There are a lot of different ways of fingerprint image segmentation, including: threshold segmentation method, variance method, and directivity diagram segmentation method. In recent years, an interactive image processing is rising as a new research method, and there is much actual application in medical treatment. Some scholars believe that the unsupervised segmentation methods can not meet the demand of the people, so joining the interactive process of human will be needed. There are several powerful interactive segmentation methods: graph cut [3], grab cut [4] and random walker [5]. They all need users' involvement to achieve high quality segmentation effect. Although the interactive image segmentation method is convenient, but to the texture image segmentation the results are not always good. So it is not suitable for fingerprint recognition for underground miners. Using directivity diagram algorithm in fingerprint image segmentation takes full advantage of the direction information of fingerprint, which has strong anti-interference ability and the automatic isolation effect on adhesion in fingerprint image. This algorithm also has automatic connection function on fracture in fingerprint image, which is the advantage of directivity diagram algorithm. But the directivity diagram algorithm also has its defects and shortcomings. When dealing with non effective area of fingerprint image, the image can not be segmented accurately because there is no effective direction of non effective area. The directivity diagram algorithm will fail at this time. This paper presents an improved method. This algorithm combines the direction partitioning and variance segmentation, which has improved the segmentation of fingerprint image to a great extent.

This paper uses $9 * 9$ direction template to perform segmentation. What is shown in Figure 3.1.2. is the $9 * 9$ direction map template. The directivity diagram segmentation method separates the foreground and background of the fingerprint. The size of the selected template here is $9 * 9$. The center of the template is the quasi point. Starting from the horizontal position, we can determine a direction every $\pi / 8$, and calculate the DI of every direction and then compare the DI of each direction, finding the minimum value of DI. We can carry out mean value calculation through the segmentation flow chart in Figure 3.1.1, and then calculate the variance of the image blocks using the formula in Figure 3.1.2. The segmentation process is shown in Figure 3.1.1. 


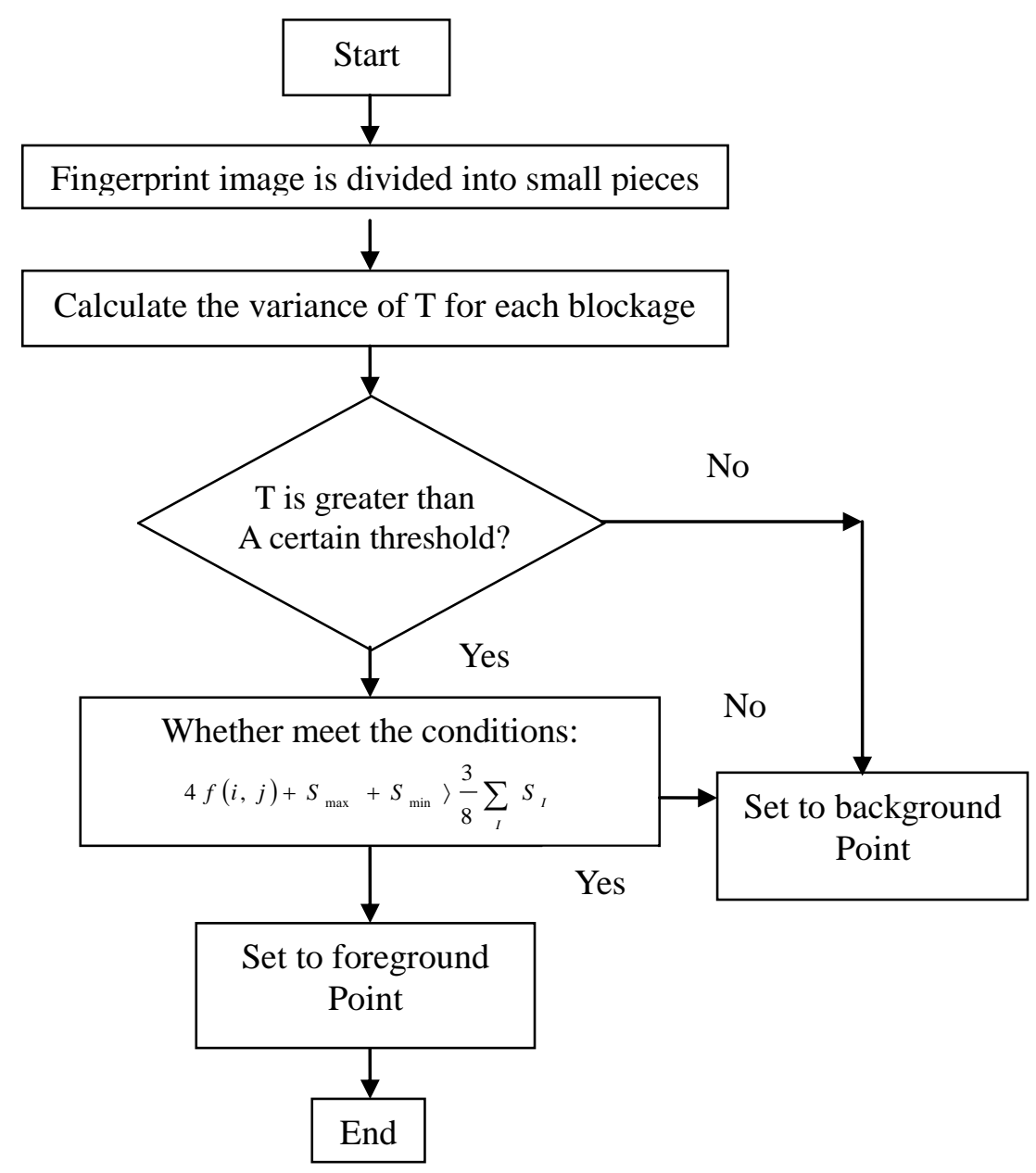

Figure 3.1.1. Flow Chart of Segmentation

\begin{tabular}{|l|l|l|l|l|l|l|l|l|}
\hline 6 & & 5 & & 4 & & 3 & & 2 \\
\hline & & & & & & & & \\
\hline 7 & & 6 & 5 & 4 & 3 & 2 & & 1 \\
\hline & & 7 & & & & 1 & & \\
\hline 0 & & 0 & & & & 0 & & 0 \\
\hline & & 1 & & & & 7 & & \\
\hline 1 & & 2 & 3 & 4 & 5 & 6 & & 7 \\
\hline & & & & & & & & \\
\hline 2 & & 3 & & 4 & & 5 & & 6 \\
\hline
\end{tabular}

Figure 3.1.2. Directivity Diagram Template 


$$
D_{I}=\sum_{k}\left|\left(f(i, j)-f_{I}\left(i_{k}, j_{k}\right)\right)\right|
$$

$\mathrm{K}$ is the number of the point in the No.1 direction, among them:

$$
\begin{aligned}
& S_{I}=\sum_{k} f_{I}\left(\mathrm{i}_{k}, j_{k}\right) \\
& \mathrm{S}_{\text {max }}=\max \left(S_{I}\right) \\
& \mathrm{S}_{\min }=\min \left(S_{I}\right)
\end{aligned}
$$

$\mathrm{S} \mathrm{i}$ is the calculation of the pixel gray value in each direction. According to the hypothesis, the central pixel of the window is written as $f(i, j)$, and the attribute of it is determined by the formula of (3-5) and (3-6).

$$
\begin{gathered}
f(i, j)\rangle \frac{1}{32} \sum_{i=1}^{s} S_{I} \\
\left.\frac{1}{8}\left(S_{\max }+S_{\min }\right)\right\rangle \frac{1}{32} \sum_{i=1}^{s} S_{I}
\end{gathered}
$$

It can be proved by the formula (3-5) that: when the gray value of the central window's pixel $f(i, j)$ is greater than the average gray value of the pixel in this window, it will be set to foreground, or else it will be set to background. Equation (3-6) has proved that: when the gray value in the direction of Smax and that of Smin are greater than the average gray value in this window, it will be set to foreground, or else it will be set to background. If the formula (3-5) and (3-6) are combined, we can draw the decision condition (3-7). If the decision condition (3-7) is established, then the point (i,j) is the foreground, or else it is the background.

$$
\left.4 f(i, j)+S_{\mathrm{max}}+S_{\mathrm{min}}\right\rangle \frac{3}{8} \sum_{I} S_{I}
$$

In order to make the segmentation of fingerprint image more smooth, first we can perform the fingerprint image segmentation using variance method, then we can use the above described decision condition. This paper puts forward an algorithm which combines variance partitioning and directivity diagram segmentation. When this algorithm performs segmentation on the fingerprint gray image, we can obtain a very good result of segmentation, which can get ready for the binarization of the fingerprint image in the next step. It can be proved that the segmentation method proposed in this paper feasible.

\subsection{The Binarization of the Fingerprint Image}

The aim of binarization is used for the thinning of an image, thereby reducing the complexity of feature extraction and matching calculation. In all kinds of papers and literatures, there are many articles about the fingerprint enhancement [6], but they do not fundamentally solve the problem of the characteristic loss in binarization. For the processing of the fingerprint image binarization, the first thing to do is the select of threshold. Different fingerprint image has different gray series. Even in the same image, the brightness of each part is always different. Therefore setting a threshold is essential to binarization.

According to the application range of the threshold, the binarization method can be divided into the overall threshold method, local threshold method and dynamic threshold method [7]. Local threshold method determines the threshold of binarization according to the gray value of the pixel and the local gray characteristics around the pixel. The dynamic threshold method 
depends on not only the gray value of the pixel and that of the pixels around it but the information about the position of the pixel [8]. The overall threshold method refers to using one threshold in the process of binarization. In the two kinds of methods about the threshold of pixel in an image, although the global threshold algorithm is simple and easy to implement, this algorithm is not suitable for the occasions of large noise. Although the local threshold algorithm can adaptively select the threshold according to the gray level, having greater flexibility, but the speed of the algorithm is slow and this method may lead to broken strokes and artifact problems. Therefore, the selection of threshold of an image is so difficult to have a universal method. The threshold is usually set according to experience and other factors [9]. You must select different method according to different specific environment. In the fingerprint recognition of coal mine workers, the accuracy is the first important thing. This paper uses the improved binarization method to perform the operation of binarization on fingerprint image after segmentation, which is based on the improvement of global iterative method.

Global iterative method is based on the idea of approximation. In global iterative method, an image is composed of the target and the background. In the gray level histogram, the target and the background all form into the peaks. The lowest point between the two peaks is the threshold of binarization in an image. The principle of the algorithm is as follows: Select an initial threshold, and its value is taken as the mean value of the maxim gray value and the minimum gray value of the image. According to the threshold the image can be divided into the target and the background. Then using the mathematical expectation of the target and the background as the new threshold, perform the binarization on the image again. Thus iterate the new threshold again and again. When the threshold is no longer changes, stop the iteration. Usually we iterate several times to reach steady state. In this paper, the binarization method which is based on the improved global iterative method can meet the the requirements of accuracy. The following is the illustration of the improved binarization method.

For a size of $m^{*} m$ image, if the pixel is $f(x, y)$ and the gray value of it is also $f(x, y)$, then the average gray value of this image is

$$
T=\frac{\sum_{i=0}^{M-1} \sum_{j=0}^{M-1} f(i, j)}{M \times M} .
$$

According to the value of $\mathrm{T}$, all the pixels of the image can be divided into two categories. The set of pixels which is less than or equal to $\mathrm{T}$ is set to $\mathrm{S} 1$, and the set of pixels which is larger than $\mathrm{T}$ is set to $\mathrm{S} 2$. Calculate the mean value of the pixels in S1 and S2 respectively, and we call them $\mathrm{T} 1$ and $\mathrm{T} 2$.

$$
T 1=\frac{\sum_{i=0}^{T} i n i}{\sum_{i=0}^{T} n i} \quad T 2=\frac{\sum_{i=T+1}^{T} i n i}{\sum_{i=T+1}^{T} n i}
$$

Through observing the fingerprint image we can find that: the prospect region has lower mean value because it contains ridge and valley lines of fingerprint, making the variance of the fingerprint image larger. On the contrary, the background region has larger mean value and smaller variance. Then in the interval [T1, T2], we can use the threshold segmentation algorithm to search the optimal threshold for segmentation, thus avoiding the search for the optimal threshold in the whole gray level.

In this paper, we use the improved binarization algorithm. This algorithm is simple, easy to 
programming. But the directional information of fingerprint must be fully taken into account, otherwise the foreground effect of fingerprint may not be ideal. So in the condition of combining the variance partitioning method and directivity diagram segmentation method, we can obtain the ideal binarization effect. It can be considered that the binarization algorithm proposed in this paper is feasible here.

\subsection{The Thinning of the Fingerprint Image}

The position of minutiae and the relative position between minutiae are very important. Although there are nearly 80 minutiae in each fingerprint, if we can identify a dozen of minutiae, it will be enough for fingerprint recognition. The most common thinning methods are listed as follows: Hilditch thinning methods, OPTA thinning methods, Sherman thinning methods, E.S.Deutsch thinning method and so on. Using different refinement methods, the refined results will vary. This paper uses the Sherman thinning methods and OPTA thinning methods, and these two thinning algorithm are the most commonly used. Therefore, this paper adopts Sherman thinning methods and OPTA thinning methods for fingerprint image processing after binarization. There is no improvement of the above methods in this paper.

The Sherman tinning algorithm in this paper can be described as: the central element of a $3 * 3$ window is placed on a black pixel which is on the edge of the black and white. In each position of the window, the method changes the color of the central element of the window into white, unless this will remove the only black pixel connecting two non adjacent black pixels which is shown in Figure 3.3.1, or the center of the window only has one black field which is shown in Figure 3.3.2. Apply the rule of thinning of pattern circularly to the fingerprint image, until the process of tracking profile no longer leads to the situation of black pixels becoming white pixels.

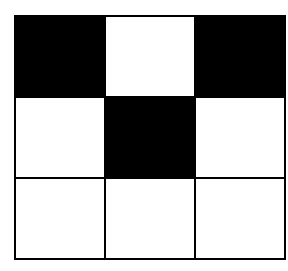

Figure 3.3.1

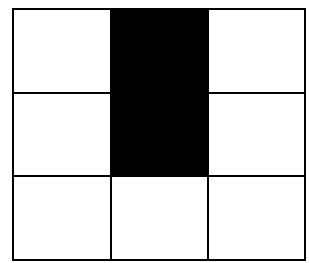

Figure 3.3.2

After using the tinning algorithm to process the fingerprint image, it turns into the streak line image and the ridge is clear and smooth after thinning, meeting the basic characteristics that a refined image should have.

OPTA tinning algorithm is a typical template matching tinning algorithm. Extract ten adjacent pixels surrounding one pixel and compare them with 8 eliminating templates and 2 reserved templates in order to judge whether the central pixel would be.

\section{Performance Test}

During operation, the first step is pressing the button 5 twice, switching to the fingerprint input mode. And then press button 4 to input fingerprint. Press the fingerprint such as the right hand thumb, then the LCD display the recording has succeeded. Then record the fingerprint again. Press the fingerprint on the sensor and the LCD display the recording has succeeded and the number of fingerprint such as 001, the buzzer sounds once at the same time. Press button 5 to switch to the fingerprint recognition mode, and then press the right thumb on the sensor. The LCD display the matching has succeeded and the number of 
fingerprint - 001. If you press the left thumb or other people's thumb, the LCD will display the matching is not successful, and the buzzer will sound three times. The scheme selects 200 people randomly for the test, and these people are divided to 20 groups. The fingerprint of each people will be recorded twice, and we record the same finger in each group. The results of the test are shown in Table 1.

Table 1. The Performance of Fingerprint Identification System

\begin{tabular}{|c|c|c|c|c|}
\hline Testers & Fingerprint & Mode & Display & The number of fingerprint \\
\hline $001-010$ & The left hand thumb & Record & Successful recording & $001-010$ \\
\hline 011-020 & The left hand thumb & Record & 011 do not display & $012-020$ \\
\hline $021-030$ & The right hand thumb & Record & Successful recording & $021-030$ \\
\hline 031-040 & The right hand thumb & Recognition & $\begin{array}{l}\text { Successful recognition } \\
\text { Do not match }\end{array}$ & 031-040 \\
\hline 041-050 & The left hand thumb & Recognition & $\begin{array}{c}\text { Successful recognition } \\
\text { Matching }\end{array}$ & 041-050 \\
\hline $051-060$ & The left hand thumb & Record & Successful recording & $051-060$ \\
\hline 061-070 & The right hand thumb & Recognition & $\begin{array}{c}\text { Successful recognition } \\
\text { Matching }\end{array}$ & 061-070 \\
\hline 071-080 & The right hand thumb & Record & Successful recording & 071-080 \\
\hline $081-090$ & The left hand thumb & Record & Successful recording & 081-090 \\
\hline 091-100 & The left hand thumb & Recognition & $\begin{array}{l}\text { Successful recognition } \\
\text { Do not match }\end{array}$ & 091-100 \\
\hline $101-110$ & The right hand thumb & Record & Successful recording & $101-110$ \\
\hline $111-120$ & The right hand thumb & Record & Successful recording & $111-120$ \\
\hline $121-130$ & The left hand thumb & Record & Successful recording & $121-130$ \\
\hline $131-140$ & The right hand thumb & Record & Successful recording & $131-140$ \\
\hline $141-150$ & The right hand thumb & Recognition & 150 do not recognize & $141-149$ \\
\hline $151-160$ & The left hand thumb & Record & Successful recording & $151-160$ \\
\hline $161-170$ & The right hand thumb & Record & Successful recording & $161-170$ \\
\hline $171-180$ & The right hand thumb & Record & Successful recording & $171-180$ \\
\hline $181-190$ & The right hand thumb & Record & Successful recording & $181-190$ \\
\hline $191-200$ & The left hand thumb & Record & Successful recording & $191-200$ \\
\hline
\end{tabular}

\section{Conclusion}

The system uses TMS320VC5509 to complete the fingerprint image acquisition and the matching function. There is some improvement in algorithm, and the accuracy of fingerprint identification is improved, which effectively enhance the operational speed of the system. Internal recognition time using this algorithm is less than one second, which is fully able to meet the need of the real time work. Two evaluation index of the fingerprint: False Accept Rate (FAR) and False Reject Rate (FRR) are both less than or equal to $1 \%$. It is suitable for the recognition of underground coal mine workers, and it can be efficient, accurate, having good value of practical application.

\section{Acknowledgements}

At the end of the paper, we thank sincerely, with great respect, to the leaders of education department of Heilongjiang province who trust us during our research and give us a chance to study the fingerprint identification system for underground miners. This is a meaningful work. We also thank headmaster Mr. Ma li-qun, and dean MS Yao zhong-ming, who also give care and support during our research. 


\section{References}

[1] Zhangkai and Xuefei, "Intelligent Queuing System Based on Fingerprint Recognition”, Electronic Device, vol. 36, no. 299, (2013).

[2] Lijianpo, "Wireless Fingerprint Attendance System Based on DSP", Process Automation Instrumentation, vol. 33, no. 29, (2012).

[3] Y. Boykov and M. P. Jolly, "Interactive graph cuts for optimal boundary and region segmentation of objects in n-d images", Proceedings of the International Conference on Computer Vision, (2001) July 10-12, Vancouver, Canada.

[4] C. Rother and V. Kolmogorov and A. Blake, "Grab cut- interactive foreground extraction using iterated graph cuts”, Proceeding ACM Siggraph, (2004) August 15-19, London, UK.

[5] L. Grady, "Random Walks for Image Segmentation", IEEE transactions on Pattern Analysis and Machine Intelligence, vol. 28, no. 11, (2006).

[6] T. K. Shih and C. Rong-chi, L. Ling-chen, "Adaptive digital image in painting", Proceedings of the 18th Intermational Conference on Advanced Information Networking and Applications, (2004) October 21-25, New York, USA.

[7] "Huwei”, Digital Signal Processing, Tsinghua University Press, Beijing, (2003).

[8] Tianzijun and Liuyi, "Global threshold binarization method based on log algorithm edge detecting", China measurement technology, vol. 33, no. 6, (2007).

[9] J. Ravi and K. B. Raja and K. R. J. Venugopal, "Fingerprint Recognition Using Minutia Score Matching", vol. 1, no. 2, (2009).

[10] Youxine, "Fingerprint information extraction research based on the orientation and dynamic threshold", Computer Simulation, vol. 29, no. 8, (2012).

Project name: Study of underground personnel positioning method based on the background of the weak signal

Project number: 12541873

Project unit: the education department of heilongjiang province

\section{Authors}

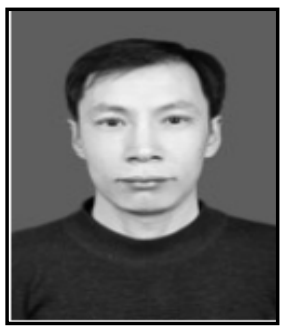

ZHU Heng-jun, Male, born in 1969, April, Qiqihaer City, heilongjiang province, associate professor, master. Teacher of communication \& electronic engineering college of Qiqihar university, heilongjiang computer association, heilongjiang electronics association.

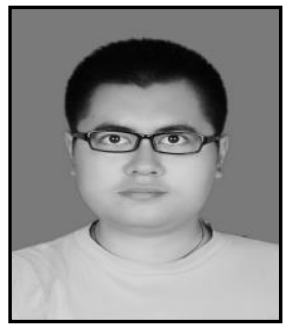

Guo Ying, Male, born in 1987, January, Nanjing City, jiangsu province, master. Student of communication \& electronic engineering college of qiqihar university. 